\title{
La elaboración de materiales bibliográficos para la formación del profesor universitario
}

\author{
Carlos Martínez-Mollineda, Viviana Hernández-Fernández y Mairelys Torres-Falcón
}

\begin{abstract}
RESUMEN
En el presente artículo, se expone parte de una investigación que se llevó a cabo en el Centro de Estudios de Dirección Empresarial (CEDE) de la Universidad Central "Marta Abreu" de las Villas, Cuba. Tiene como objetivo proponer una nueva perspectiva de aprendizaje y adquisición de competencias para alentar a los profesores a elaborar los materiales bibliográficos básicos y complementarios que se utilizan en la docencia universitaria, como soporte clave de los programas de estudios de cada carrera. Concretamente, se ofrece un proceder metodológico para estimular la elaboración de materiales bibliográficos en los departamentos docentes, resultado de una investigación cualitativa realizada en un contexto universitario. Además, se presenta el marco conceptual sobre el tema y se ilustran resultados prácticos alcanzados mediante la aplicación del proceder metodológico.
\end{abstract}

Palabras clave: proceder metodológico, materiales bibliográficos, universidad, formación, Cuba.

Carlos Martínez-Mollineda carlosm@uclv.cu Cubano. Doctor en Ciencias Pedagógicas, Universidad de Ciencias Pedagógicas "Félix Varela Morales", Cuba. Profesor titular de Administración y Gestión Empresarial, y Jefe de la disciplina, Centro de Estudios de Dirección Empresarial, Universidad Central "Marta Abreu", Las Villas, Cuba. Temas de investigación: preparación de directivos en desarrollo local, dirección educacional.

Viviana Hernández-Fernández

Cubana. Doctora en Ciencias Pedagógicas, Universidad de Ciencias Pedagógicas "Félix Varela Morales", Cuba. Profesora titular, Centro de Estudios de Dirección Empresarial, Universidad Central "Marta Abreu", Las Villas, Cuba. Temas de investigación: docencia educacional.

\section{Mairelys Torres-Falcón}

Cubana. Doctora en Ciencias Pedagógicas, Universidad de Ciencias Pedagógicas "Félix Varela Morales", Cuba. Profesora titular, Centro de Estudios de Dirección Empresarial, Universidad Central "Marta Abreu", Las Villas, Cuba. Temas de investigación: superación de directivos y su impacto en el desarrollo local sustentable, docencia educacional. 


\title{
A elaboração de materiais bibliográficos para a formação do professor universitário
}

\section{RESUMO}

No presente artigo, se expõe parte de uma pesquisa que se realizou no Centro de Estudios de Dirección Empresarial (CEDE) da Universidad Central "Marta Abreu" de las Villas, Cuba. Tem como objetivo propor uma nova perspectiva de aprendizagem e aquisição de competências para alentar aos professores a elaborar os materiais bibliográficos básicos e complementares que se utilizam na docência universitária, como suporte chave dos programas de estudos de cada carreira. Concretamente, se oferece um proceder metodológico para estimular a elaboração de materiais bibliográficos nos departamentos docentes, resultado de uma pesquisa qualitativa realizada em um contexto universitário. Além do mais, se apresenta o marco conceitual sobre o tema e se ilustram resultados práticos alcançados mediante a aplicação do proceder metodológico.

Palavras chave: proceder metodológico, materiais bibliográficos, universidade, formação, Cuba.

\section{The elaboration of bibliographic materials for the formation of the university professor}

\begin{abstract}
In the present article, part of an investigation that was carried out in the Center of Studies of Business Management (CEDE) of the Central University "Marta Abreu" of Las Villas, Cuba is exposed. Its objective is to propose a new perspective of learning and acquisition of competences to encourage teachers to elaborate the basic and complementary bibliographic materials that are used in university teaching, as a key support of the study programs of each career. Specifically, a methodological procedure is offered to stimulate the elaboration of bibliographic materials in the teaching departments, the result of a qualitative research carried out in a university context. In addition, the conceptual framework on the subject is presented and practical results achieved through the application of methodological procedure are illustrated..
\end{abstract}

Key words: methodological procedure, bibliographic materials, university, training, Cuba.

Recepción: 07/01/16. Aprobación: 08/12/17. 


\section{Introducción}

La educación superior en el mundo debe responder a los patrones internacionales y esencialmente a la dinámica de los procesos de desarrollo local y regional (Tünnermann, 2006). Para lo anterior, resulta indispensable "fortalecer la cultura del claustro sobre las ciencias de la información, la infometría, los procesadores bibliográficos y las publicaciones en general" (Alarcón, 2015: 18), lo cual contribuye en el proceso de enseñanza-aprendizaje a la generación y aplicación de conocimientos científicos y tecnológicos actualizados.

Fortalecer la cultura del profesor universitario contemporáneo requiere prepararlo para generar, investigar, organizar, coordinar, compartir, difundir y aplicar un conjunto de prácticas con sus estudiantes que den respuesta a las crecientes demandas del desarrollo y evitar que la educación se convierta en una prisión de la mente, donde las disciplinas curriculares se repitan sucesivamente (Betto, 2015).

Esta preparación de los profesores debe permitir abordar de manera actualizada los contenidos de sus programas de estudio, el rediseño de los modelos académicos, la elaboración de estrategias pedagógicas, la transdisciplinariedad y la complejidad de los currículos de las carreras porque para concebir las diferentes formas de organización de la enseñanza ha de tenerse presente que:

- Los estudiantes universitarios pueden desarrollar al máximo sus habilidades digitales, pues cuentan con la tecnología necesaria que va desde la universidad en la nube, hasta la llamada aula invertida (flipped classroom) (Bergmann y Sams, 2012). Estas habilidades constituyen herramientas con las que se puede potenciar el aprendizaje del alumnado y captar su atención (García-Barreras, 2013).

- La velocidad y cantidad de conocimientos que actualmente se generan no garantizan que en el proceso de impresión de libros se mantenga la actualidad.
- Los elementos personales que intervienen en el proceso de enseñanza-aprendizaje han cambiado el panorama del aprendizaje.

- Los medios para trabajar en la clase van de la palabra a la imagen, del libro a la información en la red electrónica, de la búsqueda individual al trabajo colectivo (Díaz-Barriga, 2011).

- Es necesario considerar a los medios y las tecnologías de información y comunicación (TIC) como un medio de enseñanza en las aulas, con su respectiva renovación didáctica y cambios en la concepción de la Educación (Medina y Ballano, 2015).

Esta realidad exige al profesor universitario el cambio y la innovación durante su actividad pedagógica profesional, para ser el protagonista de la enseñanza, el [cual, orienta, facilita y exige el trabajo de aprender, ayudando al estudiante a descubrir, investigar y realizar un autoaprendizaje, simultáneamente con la incorporación de valores y virtudes, a través de una educación creativa y motivada] (Morales, 2004: 4).

Entonces, un profesor universitario debe poseer amplios conocimientos teóricos y prácticos aplicables a la solución de problemas vitales recurrentes para realizar su trabajo al máximo de sus competencias, pues esto potencia y posibilita el desarrollo de interpretaciones compartidas y facilita acciones concertadas (Peró, 1990).

En este sentido, existe consenso en que, para lograr un aprendizaje renovado, los estudiantes han de contar con una bibliografía actualizada. Sin embargo, en un estudio realizado por los autores en la Universidad Pedagógica "Félix Varela Morales", se observan las siguientes regularidades en la práctica:

1. El profesor se basa aún en el libro de texto para guiar la toma de decisiones curriculares e instruccional durante la actividad docente.

2. Insuficiente información elaborada por el profesor en formato textual para los estudiantes. 
3. Los libros de textos que se utilizan, en su mayoría no son actualizados.

4. El seguimiento a las interacciones del contenido es difícil debido a la gran cantidad de información que hay que leer.

5. Pobre uso de materiales digitales creados por el profesorado.

El análisis detallado de estas insuficiencias permitió determinar que los profesores universitarios no siempre cuentan con una bibliografía actualizada para impartir la docencia. Por ello, iniciamos un estudio a partir de la definición del siguiente problema profesional: ¿Cómo puede el profesor actualizar la bibliografía a utilizar en la docencia universitaria para elevar la calidad del proceso de enseñanza-aprendizaje?

El objetivo del trabajo radica en proponer una nueva perspectiva de aprendizaje y adquisición de competencias para alentar a los profesores a elaborar los materiales bibliográficos básicos y complementarios que se utilizan en la docencia universitaria, como soporte clave de los programas de estudios de cada carrera.

\section{Marco conceptual sobre la actualización de los materiales bibliográficos necesarios en la docencia universitaria Acercamiento conceptual a lo que se conoce como materiales bibliográficos}

En el mundo y en especial en América Latina las universidades requieren fuertes renovaciones y transformaciones en sus concepciones, pues en muchos países predomina una fuerte influencia academicista y tecnocrática, con pobre producción científica que no responde a las necesidades de sus naciones, que conviven en una sociedad global, tecnológica y del conocimiento.

Para realizar renovaciones y transformaciones, es esencial un proceso de mejoramiento e innovación, en especial, perfeccionan las condiciones de la enseñanza y del aprendizaje, donde los medios de enseñanza ocupan un lugar significativo porque:

- Constituyen la base sobre la cual los estudiantes asimilan la realidad objetiva desde el punto de vista práctico e intelectual.

- Permiten interactuar mediante diferentes operaciones con el objeto del estudio.

- Proporcionan asequibilidad y objetividad al contenido a impartir, al permitir una mejor organización científica del proceso de enseñanza-aprendizaje.

- Facilitan la intercomunicación entre el estudiante y los profesores para favorecer la comprensión de ideas y conocimientos.

Los medios de enseñanza [constituyen un sistema de materiales docentes y de recursos tecnológicos destinados a apoyar las actividades presenciales y la autopreparación de los estudiantes; [...] como herramientas pedagógicas esenciales para facilitar el proceso de enseñanza-aprendizaje] (Horruitinier, 2006: 150).

Dentro de los medios de enseñanza se encuentran los materiales bibliográficos textuales que no han sido profundamente abordados en la literatura científica. Los autores no encontraron una definición avalada científicamente al respecto, pero consideran que se refieren a aquellos materiales impresos o digitales que los profesores pueden elaborar, como son: folletos, material de estudio, cuadernos de trabajo, monografias, compendios de notas del profesor, compendios bibliográficos, entre otros.

Todos estos materiales, una vez confeccionados, pueden ser puestos a disposición de los estudiantes, a través de diferentes vías como la red interna de la universidad y el aula virtual, entre otras, e incluirse en calidad de textos complementarios y formar parte de las colecciones existentes en las bibliotecas o centros de información científico-técnica de las universidades. 
Los materiales bibliográficos han evolucionado con el transcurso del tiempo, y hoy es posible clasificarlos en: tradicionales o actuales. Los primeros se refieren a los que históricamente se han utilizado como el libro de texto, mapas, esquemas; los actuales están relacionados con el uso de la multimedia que ha revolucionado los medios hasta límites inimaginables. Ambos tipos son indispensables en el proceso de enseñanza-aprendizaje porque:

- Facilitan la efectividad del proceso de asimilación de los contenidos a recibir por los estudiantes y estimulan el aprendizaje.

- Influyen en convertir a los estudiantes en participantes directos del proceso de enseñanza- aprendizaje.

- Contribuyen a ahorrar tiempo y energías durante el proceso de enseñanza- aprendizaje.

- Permiten la actualización del contenido que aparece en los libros de textos.

Para lograr que se elaboren los materiales bibliográficos en un departamento docente, se requiere capacitar a los colectivos pedagógicos y reforzar en ellos la colaboración, el colectivismo y la disposición de poner en función del beneficio de todos los resultados de su trabajo académico individual.

\section{Necesidad y posibilidad de que los profesores universitarios escriban sus propios materiales bibliográficos}

La universidad de hoy reclama profesores con un saber científico y capacidades para resolver problemas con la información científico-técnica, para el perfil de formación del egresado que se aspira, lo que requiere un análisis de las necesidades bibliográficas de los alumnos y su solución mediante la presentación de materiales a utilizar en cada carrera. Es oportuno aclarar que nunca un material bibliográfico va a sustituir a los libros de textos, pues la función del material bibliográfico es actualizar y complementar determinados contenidos.
Es importante que los profesores universitarios escriban sus propios materiales bibliográficos porque:

- Juegan un papel fundamental en la formación del profesional universitario, ya que el profesor es un mediador e intérprete entre la teoría y la práctica, por tanto, al escribir las bibliografías que necesitan los alumnos les facilitan el acceso a la información y a la comunicación, añadiendo ventajas a la hora de representar y experimentar la realidad.

- Propician el aprendizaje autónomo, cooperativo y la flexibilidad del currículum, así como otras mejoras pedagógicas que influyen en la formación profesional y académica mediante bibliografías actualizadas y funcionales.

- Permiten a los estudiantes la utilización de recursos bibliográficos ajustados a sus realidades, lo que facilita ampliar ideas o asociar conceptos, hacer referencia a otros documentos que tratan el mismo tema, o asociar unas temáticas con otras, creando lo que se conoce como "Circulos de conocimientos" (Mujeres, 2013).

- Facilitan la creación y actualización de contenidos que provocan una realidad bibliográfica renovada para la adquisición de nuevas experiencias y el desarrollo de habilidades.

- Contribuyen a la formación de profesionales de manera más flexible y actualizada, al resumir y sintetizar la información disponible; distribuirla acorde al diagnóstico de los estudiantes y eliminar o modificar el conocimiento obsoleto.

- Propician el desarrollo de habilidades necesarias para formar en los estudiantes la capacidad de seguir aprendiendo y desenvolverse con agilidad en su entorno una vez egresados.

De lo anterior se deduce que escribir materiales bibliográficos por el profesorado es tan importante como el proceso de edición de nuevos libros, pues constituyen un mecanismo de comunicación entre 
profesores y estudiantes. Su elaboración requiere de una adecuada coherencia didáctica, haciendo que éstos no resulten simples textos escritos o electrónicos, sino recursos adecuadamente organizados y temporalizados, donde exista un equilibrio entre el aprendizaje, la ayuda para el estudio, las actividades prácticas y los sistemas de evaluación eficaz.

Los profesores de las universidades de hoy están emplazados a crear las condiciones para que los estudiantes desarrollen al máximo su capacidad profesional, a través del proceso de enseñanzaaprendizaje, pues según Bain (2006), los mejores profesores universitarios crean el llamado entorno para un aprendizaje crítico natural, en el que incluyen las destrezas y la información que quieren enseñar mediante trabajos que los estudiantes encontrarán fascinantes, auténticos y con tareas que les motivarán a repensar sus supuestos y a examinar sus modelos mentales de la realidad.

Las posibilidades para que los profesores universitarios escriban sus propios folletos están dadas de manera general en las universidades, donde existen las tecnologías de la información y la comunicación para proveer a sus alumnos de las herramientas bibliográficas que se requieren en el siglo XXI. Los colectivos pedagógicos de estas casas de altos estudios están formados por académicos que tienen experiencia en escribir diferentes textos, ya sea para eventos, artículos para revistas, tesis, conferencias, entre otros trabajos; sólo se necesita un breve entrenamiento que facilitará al profesor, a partir del conocimiento particular, combinar e integrar los textos sistémicamente en materiales elaborados para fines docentes.

\section{Nueva perspectiva de aprendizaje y adquisición de competencias para escribir los materiales bibliográficos}

Encontrar una perspectiva de aprendizaje para que los profesores adquieran las competencias requeridas para escribir materiales bibliográficos, plantea un problema teórico y metodológico, pues debe demostrarse cómo éstos, en la dinámica de su trabajo cotidiano, pueden motivarse y lograr escribir materiales bibliográficos.

La solución del problema antes planteado, requirió del estudio de la evolución que han tenido las tecnologías, tales como: las precisiones de la neo-competencia laboral, así como la formación por competencias, tema que en la Unión Europea tuvo cierto impacto debido a la necesidad de reconocer en diferentes países las habilidades que se exigen para determinadas labores y la necesidad de hacer coincidir el trabajo sinérgico entre competencia personal y organizacional (Díaz-Barriga, 2005); (Vega, 2016).

$\mathrm{El}$ enfoque por competencias tiene en cuenta las teorías del aprendizaje contextualizado en el aprendizaje de oficio y activo. A partir de esto se han aplicado diversos modelos de formación por competencia en el nivel universitario (Tobón, 2006).

En este sentido, Taca (2011) señala que dentro de las competencias esenciales de los docentes en el contexto actual está "producir materiales educativos acordes a diferentes contextos para favorecer los procesos de enseñanza y aprendizaje". También se tiene en cuenta la Teoría General de Sistemas (Bertalanfly, 1954), esencialmente un concepto de "sistema" ofrecido por Samoura (1999: 20), quien plantea que [el sistema no es solamente un conjunto de componentes y propiedades cuyas relaciones e interacciones engendran una nueva cualidad integradora, sino también el carácter funcional o la funcionalidad y la interfuncionalidad entre los componentes integrantes].

Además, Samoura precisa que la principal particularidad definitoria de un sistema es la existencia de una cualidad resultante, generada por las relaciones e interacciones entre los componentes del sistema, fruto de la naturaleza sistémica de esas relaciones e interacciones. Esta sustentación orientó a los autores de este artículo a plantear que la preparación de los profesores para escribir los materiales bibliográficos podía realizarse si se logra conformar un sistema 
para el desarrollo de competencias, mediante determinados procederes que automaticen un conjunto de pasos interrelacionados, que describan una secuencia lógica del proceso a través de componentes y acciones necesarias para obtener la formación de competencias requeridas en el profesorado que labora en el contexto universitario actual.

En la literatura científica consultada se constató que el término procederes, no es ampliamente abordado teóricamente. Sin embargo, el vocablo procedimiento tiene una profunda utilización en la bibliografía pedagógica y, a juicio de los autores, las palabras procederes y procedimientos tienen significados similares, si se tiene en cuenta que los procedimientos son aquellos apoyos al profesor para determinadas actividades docentes, fundamentalmente las relacionadas con la enseñanza y el aprendizaje, pues actúan como elementos estratégicos que pueden ser utilizados para la conformación de terminadas estrategias de aprendizaje.

Por tanto, entendemos por procederes metodológicos aquellos procedimientos que actúan como complemento de los métodos de enseñanza, o sea, son "herramientas" que permiten alcanzar objetivos, mediante acciones o actividades, a partir de las características del contenido, que orientan cómo accionar para lograr el éxito en una determinada actividad (Silvestre y Zilberstein, 2000).

Los procederes metodológicos se elaboran específicamente para una determinada actividad de aprendizaje y no deben ser generalizados a otras diligencias. Por ejemplo, un proceder para dirigir una institución educativa, no debe utilizarse mecánicamente para dirigir una empresa.

Esta especificidad de los procederes metodológicos permite establecer algunas particularidades de este tipo de herramienta metodológica, por ejemplo: los procederes metodológicos se diseñan para conseguir un objetivo determinado; demandan determinar con antelación el área donde se van a utilizar; requieren una secuencia para su introducción; implican determinados conocimientos para realizar las acciones; demandan desarrollar habilidades para su aplicación; exigen elaborar orientaciones generales que aseguren su aplicación.

De acuerdo con los rasgos anteriores, la elaboración de un proceder metodológico debe tener una estructura que oriente al usuario en cómo utilizarlo de manera clara y precisa. Por ejemplo, debe contar al menos con los siguientes componentes integrados y coherentemente organizados: a) Objetivos, b) Principios en los que se sustentan, c) Fases para su aplicación, d) Cada fase debe contar con determinados pasos, e) Orientaciones para su utilización, f) Evaluación y g) Cualidad resultante.

Un proceder metodológico se entrena de forma sistemática en la solución de situaciones problemáticas profesionales, transitando por las etapas de la actividad para que gradualmente sea interiorizado hasta lograr aplicarse en un plano mental, con lo cual será capaz de, ante nuevos problemas, obtener resultados superiores, debido al nivel de autorregulación alcanzado. Es decir, un proceder metodológico sistematizado puede ser interiorizado desde lo externo a lo interno en forma de actividad interna mental, que es recomendable para enseñar a los profesores a escribir sus propios materiales bibliográficos.

En fin, la aplicación adecuada de un proceder metodológico puede facilitar un impacto positivo sobre el bienestar psicológico de un colectivo pedagógico universitario al proporcionar satisfacción laboral, tal como afirman Atkinson (1957) y Child (1986), pues el individuo, al alcanzar determinadas competencias, puede hacer posibles comportamientos positivos en aspectos relativos a la tarea o el rol que desempeña, así como conductas más relevantes en su desempeño en la organización a la que pertenece.

\section{Preparación de los profesores para escribir los materiales bibliográficos}

El objetivo general de este trabajo está dirigido a enseñar y estimular a los profesores universitarios a actualizar, por ellos mismos, la bibliografía a utilizar 
en la docencia universitaria, mediante el incremento de los conocimientos, el desarrollo de las habilidades necesarias y el cambio de modos de actuación en cuanto a la escritura de los materiales bibliográficos por parte de los profesores.

\section{Método}

Teniendo presente el objetivo general descrito anteriormente, se formuló la siguiente hipótesis de investigación: si los profesores del Departamento de Dirección Científica Educacional incrementan la motivación, amplían los conocimientos y desarrollan habilidades para escribir sus propios materiales bibliográficos, entonces se incrementa la producción actualizada de materiales bibliográficos en función de elevar la calidad de la formación del profesional universitario.

Para estudiar la hipótesis se utiliza una metodología cualitativa organizada en cuatro fases de forma sistemática y orientada a la comprensión en profundidad de un fenómeno educativo interpretado, explicado y comprendido en su propio contexto (Sandín, 2003).

\section{Fases claves de la investigación Primera fase: diagnóstico inicial}

Sobre la base de los fundamentos teórico-metodológicos expuestos antes, se realizó un diagnóstico de los profesores en el Departamento de Dirección Científica Educacional (DCE), ahora integrado al Centro de Estudios de Dirección de la Universidad Central "Marta Abreu" de Las Villas, Cuba, con un total de 15 profesores, de ellos el 60\% (9) son doctores; el $40 \%$ (6) maestros y el $86 \%$ ostenta categorías superiores (9 PT y $4 \mathrm{PA}$ ), para conocer si estaban preparados para elaborar los materiales bibliográficos para sus alumnos.

La razón fundamental para elegir este Departamento fue la adecuada composición de su claustro y porque es donde laboramos los autores de este trabajo, lo que permite realizar un seguimiento sistemático. Para profundizar en el diagnóstico se plantea la siguiente interrogante: ¿Cuáles son las fortalezas y debilidades que poseen los profesores del Departamento de Dirección Científica Educacional para escribir materiales bibliográficos en cuanto a conocimientos, habilidades y motivación?

\section{Procedimiento utilizado para realizar el diagnóstico}

La realización del diagnóstico se organizó sobre la base de tres etapas fundamentales: la selección de instrumentos, aplicación de instrumentos y la interpretación de sus resultados.

Selección de instrumentos. Los instrumentos seleccionados fueron: el análisis documental, la guía de observación, guía de entrevistas y la encuesta:

- Guía para el análisis documental: se realiza para apoyar otros métodos más directos de recogida de datos como la observación participante y la entrevista en profundidad. En el caso específico de esta investigación, se utilizó el estudio de documentos oficiales internos del Ministerio de Educación Superior (Anexo 1).

- Guía para la observación científica: permite obtener la información del comportamiento del objeto de investigación tal y como éste se da en la realidad, o sea, es una forma de obtención de información directa e inmediata sobre el fenómeno u objeto que está siendo investigado (Anexo 2). En este trabajo se aplica la observación participante para percibir el fenómeno tal y como se desarrolla, buscando obtener información más confiable, para contrastarla con las otras técnicas empleadas.

- Guía para las entrevistas: la entrevista es una técnica en la que una persona solicita información de otra, por lo tanto presupone la participación de al menos dos personas y la posibilidad de interacción verbal. En la presente investigación se llevó a cabo una entrevista semiestructurada al $100 \%$ de los profesores, con el objetivo 
de identificar las fortalezas y debilidades para la escritura de los materiales bibliográficos (Anexo 3).

- Guía de encuestas: este instrumento se aplica con el objetivo de conocer la preparación que tienen los profesores de DCE en la escritura de materiales bibliográficos para lograr una mayor y mejor formación del profesional universitario (Anexo 4).

La aplicación de instrumentos se realizó durante septiembre y octubre de 2011 y se ha dado seguimiento hasta 2015 de manera sistemática. La interpretación de los instrumentos arrojó los siguientes resultados:

\section{Fortalezas}

- El departamento cuenta con un total de 15 profesores, de ellos el 60\% (9) son doctores con experiencia, formación que facilita escribir materiales bibliográficos.

- El 100\% tiene conocimientos de la educación superior.

- El $100 \%$ domina los contenidos básicos de la disciplina integradora "Dirección o Administración".

- El $86 \%$ ostenta categorías superiores (9 PT y $4 \mathrm{PA})$; de igual forma mantienen estrecha cooperación con una valiosa red de colaboradores, tanto en la Universidad Central de Las Villas como fuera de ella.

- Un claustro preparado y con experiencia para el desarrollo de los procesos pedagógicos en la educación superior.

- La planta profesoral es utilizada en correspondencia con la categoría docente que posee cada profesor.

- Los docentes dominan la asignatura que imparten, lo que se corrobora con los resultados de las visitas e inspecciones realizadas en los últimos cinco años.

\section{Debilidades (jerarquizadas)}

- Insuficiente motivación para acometer las tareas actuales de escribir los propios materiales bibliográficos y garantizar la actualización bibliográfica de los contenidos.

- No se aprovecha al máximo la capacidad científica y creativa de los docentes para escribir los materiales bibliográficos.

- No se planifican ni se organizan, de manera sistemática y objetiva, actividades que aseguren la preparación de profesores noveles, para desarrollar habilidades en la escritura de materiales bibliográficos.

- Limitadas acciones en el plan de trabajo metodológico del centro y en la estrategia para escribir los materiales bibliográficos por los profesores.

- Ausencia de acciones en la planeación estratégica para que los profesores estén en condiciones de escribir el material bibliográfico.

- No se utilizan herramientas que orienten a los profesores sobre cómo escribir sus materiales bibliográficos.

Estas debilidades indican insuficiencias en los profesores del DCE para escribir materiales bibliográficos, lo que apunta a carencias de conocimientos que actúen como "herramientas" que promuevan cambios en la conducta de los profesores respecto a la escritura de los materiales bibliográficos actualizados para la formación del profesional universitario, lo que requiere incrementar los conocimientos, desarrollar las habilidades y reforzar la motivación.

\section{Segunda fase. Cognitiva}

La determinación de fortalezas y debilidades planteó un nuevo problema para esta investigación: la necesidad de incrementar las nociones y representaciones que los profesores poseen para elaborar materiales bibliográficos o sea, conocimientos necesarios que faciliten su escritura. 
Para resolverlo, se recurrió a una intervención educativa del Centro de Información Pedagógica (CEDIP) donde se introdujeron herramientas de análisis y reflexión para escribir los materiales bibliográficos en siete sesiones de trabajo, en las cuales se abordaron: diferentes normas bibliográficas, patrones de la escritura y redacción de los materiales científicos, la navegación en revistas; catálogos de bibliotecas, bases de datos bibliográficas, bases de datos no bibliográficas, revistas electrónicas, documentos en formatos no indizables, como documentos en PDF, Word, enciclopedias y diccionarios.

Esta segunda fase resultó significativa, ya que para escribir los materiales bibliográficos fue imprescindible conocer teóricamente cómo se escriben estos, enfatizar en las técnicas para estimular su escritura, en correspondencia con los objetivos marcados en sus programas, nociones y representaciones sobre los materiales bibliográficos, así como la metodología para la elaboración de compendios, folletos y libros.

Una vez incrementados los conocimientos en los profesores sobre la escritura de materiales bibliográficos, surge la necesidad de desarrollar habilidades para esta actividad.

\section{Tercera fase. Desarrollo de habilidades}

Para enfrentar este problema se realizaron siete sesiones de trabajo, concentrando la actividad fundamental en la escritura, en las habilidades de redacción, ortografía y fuentes de asentamiento bibliográfico, la empatía y la toma de decisiones. Resultaron muy valiosas estas sesiones porque permitieron reconocer que cualquier resultado científico diseñado para que los profesores escriban sus materiales bibliográficos, requieren de un entrenamiento táctico para el desarrollo de habilidades. Se desarrollaron actividades dirigidas a: búsqueda de información, selección de documentación en bases de datos y buscadores académicos, habilidad de argumentación, capacidad de autoevaluación, así como consolidar la habilidad de aprendizaje autónomo y de trabajo en equipo.

\section{Cuarta fase. Elevar la motivación por la escritura de materiales bibliográficos}

En la medida en que se le dio tratamiento al desarrollo de habilidades para la escritura de materiales bibliográficos en los profesores, se reforzó la motivación y voluntad que permitieran transformar sus modos de actuación ante la necesidad y reto de crear sus propios materiales bibliográficas para el fortalecimiento del proceso de enseñanza aprendizaje.

Se dedicaron cinco sesiones adicionales de trabajo para el desarrollo de la motivación, enfatizando la autoconfianza, la autoestima y la necesidad de escribir los materiales bibliográficos. Esta fase resultó clave porque cualquier resultado científico que se proponga, debe contener momentos para el desarrollo de la motivación, involucrando a los participantes en la investigación a través de la investigación-acción en:

- Transformar la situación de preparación existente para escribir los materiales bibliográficos.

- Concientizar a los profesores en la importancia de llevar a cabo, desde la propia carga docente, la labor de escribir los materiales bibliográficos necesarios.

- Proponer posibles acciones para elevar su preparación respecto a la elaboración de materiales bibliográficos por ellos mismos (profesores) para ajustar el sistema de conocimientos a las necesidades de la carrera y de los estudiantes de pregrado y posgrado, de acuerdo con el desarrollo científico técnico y el contexto de actuación del profesional universitario.

Cada una de las cuatro fases anteriormente descritas, se desarrollaron a través de actividades que tenían una estructura similar compuesta por:

Motivación: estimulación de los participantes para aumentar progresivamente la conciencia sobre la necesidad de escribir los materiales bibliográficos 
e identificar temáticas que se desarrollarían con detalle en la sesión o actividad.

Desarrollo: una vez generado un clima adecuado, se proponían dinámicas para elevar el nivel de motivación, conocimiento y disposición para que los profesores del DCE escribieran los materiales bibliográficos de los estudiantes. El trabajo se desarrollaba de modo individual, en parejas o en grupo, y se empleaban técnicas participativas.

Conclusiones: en esta fase, se dejaban 10 minutos para valorar el cumplimiento de los objetivos de la actividad.

En total, se realizaron 20 sesiones de trabajo en las que emergieron categorías y subcategorías claves para la preparación de los profesores en la escritura de sus materiales bibliográficos. Éstas se evidencian en la tabla 1 .

\section{Resultados y discusión}

$\mathrm{Al}$ aplicar la lógica del ascenso progresivo de lo abstracto a lo concreto, en función de la solución del problema planteado y el procedimiento descrito en el epígrafe anterior, fue emergiendo en la discusión un proceder metodológico para estimular la elaboración de materiales bibliográficos formados por los siguientes elementos: fundamentos pedagógicos, psicológicos y sociológicos; objetivos, principios, fases y pasos; sugerencias didácticas y su evaluación.

Los participantes consideran que para escribir materiales bibliográficos se requieren procederes metodológicos sustentados en la pedagogía, la psicología y la sociología. De esta necesidad emergieron los siguientes fundamentos:

Fundamentos pedagógicos: los procederes responden a un conjunto de exigencias pedagógicas para escribir los materiales bibliográficos que contribuyen a la materialización de la unidad entre la instrucción y educación, y entre los diferentes componentes del proceso de enseñanza-aprendizaje, así como el carácter activo, consciente y significativo, acorde con las características de los departamentos docentes y su contexto educativo. Lo anterior evidencia el cumplimiento de leyes y principios que norman y regulan dicho proceso.

Fundamentos psicológicos: los procederes persiguen movilizar la aparición de motivos intrínsecos a través de la actividad y comunicación, que a su vez promuevan significatividad de los materiales bibliográficos, implicando a los profesores en la producción de los mismos, desplegando para ello su actividad productivo-creadora, meta-cognitiva y perfeccionándose en un contexto de relaciones sociales.

Tabla 1. Categorías que surgen de las sesiones de trabajo

\begin{tabular}{|l|l|}
\hline \multicolumn{1}{|c|}{ Categorías } & \multicolumn{1}{c|}{ Subcategorías } \\
\hline Preparación de profesores & $\begin{array}{l}\text { Conocimientos } \\
\text { Valores } \\
\text { Orientación hacia la mejora }\end{array}$ \\
\hline Escribir los materiales bibliográficos & $\begin{array}{l}\text { Habilidades } \\
\text { Empatía } \\
\text { Comunicación }\end{array}$ \\
\hline Proceder metodológico & $\begin{array}{l}\text { Herramientas para la gestión } \\
\text { Métodos } \\
\text { Ciclo de acción }\end{array}$ \\
\hline
\end{tabular}

Fuente: elaboración propia. 
Fundamentos sociológicos: los procederes diseñados tienen presente el desarrollo del individuo bajo la influencia de la educación y el medio, por su contenido social, como una unidad dialéctica entre la objetivación (materialización) y subjetivación (asimilación) de los contenidos sociales.

Una vez determinados los fundamentos fue necesario precisar el objetivo de la construcción del proceder metodológico: propiciar la elaboración de materiales bibliográficos por los profesores para ajustar el sistema de conocimientos a las necesidades de la carrera y de los estudiantes de pregrado y posgrado, acordes al desarrollo científico técnico y al contexto de actuación del profesional universitario.

Se considera necesario precisar los principios que sustentan el proceder metodológico:

- Gestión del conocimiento actualizado: se refiere al proceso de creación, captura y utilización del conocimiento en materiales bibliográficos para incrementar los niveles cognitivos de los estudiantes de manera actualizada, de forma tal que se satisfagan las exigencias de los estudiantes y de la carrera.

- Almacenamiento del conocimiento: para que pueda ser utilizado el conocimiento debe hacerse explícito en depósitos funcionales, como pueden ser los materiales bibliográficos en forma dura o virtual u otros medios de almacenaje y distribución, ajustando el contenido con los nuevos criterios de la ciencia y la técnica.

- Adaptabilidad del conocimiento al contexto: lograr su creación acorde al contexto de actuación del estudiante según la carrera y la asignatura.

- Inclusión del entorno: tener presente la comunidad donde se inserta el futuro profesional universitario como fuente para la localización y adquisición de los conocimientos externos.

El proceder seguido necesitó de un orden para alcanzar el objetivo mediante diferentes fases y pasos:
1. Fase de motivación al profesorado: es la fase donde se crea conciencia de la necesidad de escribir los materiales bibliográficos, se ofrecen argumentos y se busca la manera de lograr un nivel de implicación de los profesores con la creación y producción de materiales bibliográficos; dirigida al desarrollo de la motivación enfatizando en la autoconfianza, la autoestima y la necesidad de escribir los materiales bibliográficos.

2. Fase de diagnóstico: consiste en la identificación de los profesionales con mayor nivel de conocimientos y posibilidades para escribir sus propios materiales bibliográficos en el departamento y se establecen los vínculos de colaboración; los pasos seguidos fueron: identificación de los profesionales con mayor nivel de conocimiento y experiencia en la escritura de los materiales bibliográficos en la universidad y comprometerlos con la preparación del resto de los profesores; determinación de fortalezas y debilidades del colectivo de profesores para escribir los materiales bibliográficos.

3. Fase de demostración: es la fase donde se capacita a los docentes en el puesto de trabajo para que escriban sus materiales bibliográficos, a partir de los procedimientos de cada tipo de material, las normas bibliográficas, entre otros aspectos que están relacionados con el rigor científico de los mismos de acuerdo con las exigencias y particularidades de cada carrera. Los pasos referidos fueron: a) Información inicial: se informa el objetivo y las características de la formación a adquirir durante la etapa de demostración; b) Capacitación cognitiva: dirigida a incrementar las nociones y representaciones de los profesores que le permitan escribir un material bibliográfico, mediante el estudio de los contenidos básicos; c) Desarrollo de habilidades: es un paso de entrenamiento dirigido a desarrollar un conjunto de habilidades para la elaboración de materiales bibliográficos acordes a las exigencias 
de la Educación Superior; d) Involucramiento: dirigido a incrementar la voluntad de los profesores para escribir materiales bibliográficos, condicionados por la necesidad que existe en el estudiantado universitario, donde se requiere que los participantes lleguen a las siguientes conclusiones: necesidad de trabajo en equipo, de comunicación, de motivación y entusiasmo por escribir los materiales bibliográficos, con optimismo para el triunfo, y con deseos de cambiar y de hacer las cosas diferentes.

4. Fase de generalización: es la fase en que se generaliza a todo el departamento la producción de materiales bibliográficos y comienza la producción individual o en colectivos de autores. Los pasos seguidos fueron: generalización de la escritura de materiales bibliográficos por todos los profesores del departamento; organización de los colectivos de autores; definición de las temáticas a escribir, plazos para la entrega y características de los productos.

5. Fase de controly evaluación: en esta fase se controla la calidad de los materiales elaborados y su aprobación para utilizarse en la docencia. Los pasos fueron: creación de una comisión especializada en el departamento docente para la aprobación de los materiales bibliográficos; determinación de los parámetros para la evaluación de cada material bibliográfico y su divulgación; aprobación y evaluación de los materiales bibliográficos por la comisión creada al efecto.

6. Fase de mejora continua: fase en la que se busca una dinámica continua de estudio mediante el análisis de los materiales bibliográficos elaborados, las experiencias, la satisfacción del perfil del egresado, el estudiante y la carrera en su conjunto para producir productos textuales de la más alta calidad. Los pasos seguidos fueron: evaluar la calidad de los productos elaborados teniendo presente diseño, contenidos y funcionabilidad; establecimiento de las metas y criterios de calidad para continuar con la mejora continua, fijando nuevos objetivos que mejoren los resultados anteriores de la producción de materiales bibliográficos, basándose en anteriores resultados, los datos y la experiencia.

El proceder construido requirió de algunas aclaraciones, emergiendo así las sugerencias didácticas para la elaboración de los materiales bibliográficos. Ellas son:

I. La orientación del contenido. Presentar al estudiante el contenido desde diferentes aristas, en busca de la polémica, para que el estudiante tome partido ante las situaciones dadas donde se definan con claridad las exigencias y condiciones para el análisis del contenido.

El contenido debe evidenciar la unidad entre el conjunto de requerimientos propuestos para revelar los objetivos, exigencias o intencionalidades y la estimulación al esfuerzo intelectual en el análisis del contenido.

II. Presentación de ejercicios al final de cada tema. El estudiante puede analizar el contenido y pasar a la solución de los ejercicios al finalizar el tema. Los ejercicios permiten el producto de la actividad y el intercambio en colectivo, donde se soluciona de manera individual y se seleccionan aquellas respuestas que serán demostrativas. Posteriormente se propician las condiciones psicológicas y ambientales favorables para perfeccionar los productos creados.

Esta característica de los materiales bibliográficos elaborados, hace posible comprobar cómo los estudiantes son capaces de asimilar el contenido, aplicando y reestructurando sus conocimientos a las particularidades de sus carreras.

Otras sugerencias didácticas a tener en cuenta son: variedad de enfoques sobre un mismo contenido; temas que motiven a los estudiantes; propiciar la 
reflexión individual; tener en cuenta la planificación de los temas del programa y las formas de organización del proceso de enseñanza-aprendizaje; partir del diagnóstico de los estudiantes; en la preparación de la asignatura, valorar la utilización de los materiales elaborados para poder medir la efectividad de los mismos, a fin de realizar las correcciones oportunas

\section{Principales resultados obtenidos con la aplicación de los procedimientos}

Estos procederes metodológicos fueron aplicados en el Departamento de DCE de la antigua universidad de Ciencias Pedagógicas "Félix Varela Morales" desde el 2011 hasta el 2015. Los resultados más significativos obtenidos fueron:

1. Libro de texto titulado Desarrollo histórico de la dirección del proceso de preparación y superación de los cuadros educacionales y sus reservas [ISBN 978-95913-2634-8], Editorial Pueblo y Educación, que obtuvo el Tercer Premio de Ciencia Innovación a nivel nacional.

2. Monografía sobre "Desarrollo histórico de la dirección del proceso de preparación y superación de los cuadros educacionales y sus reservas".

3. Libro: La universidad moderna y su reto mayor. Reflexiones y experiencia en latino-américa (Dirección Educacional), Alemania, Editorial Académica Española [ISBN 978-3-8484-5576-8].

4. "Manual de estudio para la gestión del Director Municipal de Educación”, presentado en el Fórum de Ciencia y Técnica del año 2012, con una opinión crítica muy favorable.

5. Compendio para estudiantes del tercer año de la carrera Pedagogía-psicología, que es utilizado como un material base en la misma carrera.

6. Un artículo denominado "El rol del profesor del departamento de Dirección Científica Educacional", en Cuadernos de Educación y Desarrollo, indexada en IDEAS-RePEc y alojada en www. eumed.net/rev/ced.

7. Compendio para el posgrado "La gestión pedagógica en la Dirección Científica Educacional".

\section{Conclusiones}

Los docentes universitarios requieren cumplir una serie de requisitos para estar acordes con las exigencias de la educación superior actual. Éstos deben saber elaborar sus propios materiales bibliográficos para integrar de manera objetiva la teoría y la práctica, y no ser simples reproductores de conocimientos obsoletos.

La preparación del profesorado universitario para escribir los materiales bibliográficos es un elemento clave en la actualización de la bibliografía y en la formación integral del profesional universitario, pues proporciona una amplia variedad de experiencias, información, materiales y posibilidades de comunicación científica y cultural que enriquecen y flexibilizan la docencia universitaria.

La calidad de la confección de los materiales bibliográficos puede estar en manos de los profesores y autoridades educativas, para ser verdaderos constructores de conocimientos y promotores de los cambios que requiere la sociedad en busca de un desarrollo sostenible. No obstante, debe tenerse en cuenta que sus beneficios pueden continuarse investigando y evaluando su impacto en el proceso de enseñanza-aprendizaje.

El proceder metodológico construido en esta investigación está compuesto por fundamentos pedagógicos, psicológicos y sociológicos, objetivos, principios, fases y pasos, sugerencias didácticas y su evaluación, dirigido a fomentar la escritura de materiales bibliográficos por los propios profesores. Puede ser generalizado en los departamentos docentes de las universidades siempre que se contextualicen a su realidad y sean cuidadosamente revisados por las comisiones científicas de cada departamento. 


\section{Referencias}

Alarcón, R. (2015), "Las ciencias de la Educación en una universidad integrada e innovadora", Congreso Pedagogía, 26 al 30 de enero, La Habana, Ministerio de Educaciòn Superior.

Atkinson, J. W. (1957), "Motivational determinats of risktaking behavior", en Psycological Review, 359-372.

Bain, K. (2006), Lo que hacen los mejores profesores de universidad, Valencia, España, Editora Palacios.

Bergmann, J. y A. Sams (2012), Flip your classroom: talk to every student in every fip, Washington, ISTE.

Bertalanfly, L. V. (1954), Teoría general de los sistemas, Nueva York, Pitman.

Betto, F. (2015) "Educación crítica y protagonismo cooperativo", Conferencia especial del teólogo y escritor brasileño Frei Betto, <http://linkis.com/ cTNcu> [Consulta: enero 2015).

Child, J. (1986), New Tecnology and developments in management organization, Berlín, T. Lupton: human factors.

Díaz-Barriga, Á. (2005) "El enfoque de competencias en la educación. ¿Una alternativa o un disfraz de cambio?", en Perfiles Educativos, <http://www.scielo. org.mx/scielo.php> [Consulta: septiembre 2015].

Díaz-Barriga, Á. (2011), "Competencias en educación. Corrientes de pensamiento e implicaciones para el currículo y el trabajo en el aula", en Revista Iberoamericana de Educación Superior, núm. 5, vol. II, pp. 3-24.

García-Barreras, A. (2013), "El aula inversa. Cambiando las respuestas a las necesidades de los alumnos", en Avances en Supevisión Educativa, núm. 19, noviembre, pp. 1-8.

Horruitiner, P. (2006), La universidad cubana: el modelo de formación, La Habana, Editorial "Félix Varela".

Medina, A. y S. Ballano (2015), "Retos y problemáticas de la introducción de la educación mediática en los centros de secundaria”, en Revista de Educación, núm. 369, julioseptiembre, pp. 134-158.

Morales, D. (2004), La Creatividad en la Educación Superior, Santo Domingo, Impresión ligera.

Mujeres, O. N. (2013), Presentación del Portal de Conocimientos, una web para el empoderamiento económico de las mujeres, e-igualdad.ne, <http://www.e-igualdad.net> [Consulta: marzo 2015].

Peró, J. M. (1990), Organizaciones: nuevas perspectivas psicosociológicas, Barcelona, PPU.

Samoura, K. (1999), Diseño de un modelo sistémico de dirección científica y metodológica de la educación fisica y el deporte, La Habana, Instituto Superior de Cultura Física.

Sandín, E. (2003), La investigación cualitativa en educación. Fundamentos y tradiciones, Madrid, McGraw Hill.

Silvestre, M. y J. Zilberstein (2000), ¿Cómo hacer más eficiente el aprendizaje?, México, Ediciones CEIDE.

Taca, D. R. (2011), "El nuevo enfoque pedagógico: las competencias", en Investigación Educativa, vol. 15, núm. 28, pp. 163-185.

Tobón, S. (2006), Formación basada en competencias, Madrid, Universidad Complutense de Madrid.

Tunnermann, C. (2006), Pertinencia y calidad de la educación superior, Guatemala, Universidad Rafael Landivar .

Vega, N. D. (2016), "Neocompetencias, nuevo enfoque de competencias laborales en Salud Ocupacional", en Revista Educación Médica Superior, <scielo.sld.cu/scielo. php> [Consulta: enero 2016]. 


\section{Anexo 1. Guía para el análisis documental}

Documentos a analizar:

- Resolución 210: "Reglamento docente-metodológico para la Educación Superior"

- Estrategia del centro de estudio (DCE) hasta el 2017.

- Plan de trabajo metodológico de la DCE.

- Plan de superación de los profesores de la DCE.

- Transformaciones para la educación superior en Cuba.

- Materiales bibliográficos elaborados por los profesores de la DCE.

Aspectos a analizar:

- Cantidad y calidad de materiales bibliográficos elaborados por los profesores de la DCE.

- Incentivos a los profesores para escribir sus propios materiales bibliográficos y garantizar la actualización bibliográfica de los contenidos.

- Capacidad científica y creativa de los docentes para escribir los materiales bibliográficos e introducir en el proceso docente educativo, de manera tal que se logre la actualización requerida.

- Planificación de actividades para la preparación de profesores, para desarrollar habilidades en la escritura de materiales bibliográficos.

- Acciones metodológicas en el plan de trabajo metodológico del DCE y en la estrategia para alcanzar motivación, conocimientos y habilidades en la escritura de materiales bibliográficos por los profesores.

- Acciones estratégicas para que los profesores estén en condiciones de escribir el material bibliográfico.

- El aseguramiento bibliográfico de la disciplina integradora de Administración en las carreras de la universidad (Ingeniería Industrial, Contabilidad y Finanzas, Economía, Telecomunicaciones, Ingeniería Eléctrica, Automática, Informática, Mecánica, Comunicación Social, Ciencias de la información, Lengua Inglesa.

- El aseguramiento bibliográfico en la red.

- Precisiones normativas en resoluciones de la educación superior cubana e internacionales. 


\section{Anexo 2. Guía para la observación}

Objetivo: analizar las deficiencias que presenta el proceso de elaboración de materiales bibliográficos por parte de los profesores de Dirección Científica Educacional (DCE) de la Universidad Central de Las Villas.

Unidad de Observación: Departamento de Dirección Científica

Variable: preparación para escribir materiales bibliográficos

\begin{tabular}{|c|c|c|}
\hline Dimensiones & Concepto & Evaluación \\
\hline Conocimientos & $\begin{array}{l}\text { Nociones y representaciones que los profesores } \\
\text { poseen para elaborar materiales bibliográficos }\end{array}$ & $\begin{array}{l}\text { A. Conocimiento alto. Cuando el conocimiento } \\
\text { expresado por el profesor demuestra que poseen } \\
\text { adecuadas nociones y representaciones a través } \\
\text { de juicios y valoraciones propias para elaborar } \\
\text { materiales bibliográficos. } \\
\text { M. Conocimiento medio. Cuando el conocimiento } \\
\text { expresado por el profesor es descriptivo, sin } \\
\text { presentar una integración lógica acerca de cómo } \\
\text { elaborar materiales bibliográficos. } \\
\text { B. Conocimiento bajo. Cuando no demuestra } \\
\text { pobres nociones y representaciones acerca de } \\
\text { cómo elaborar materiales bibliográficos }\end{array}$ \\
\hline Habilidades & $\begin{array}{l}\text { Dominio de un conjunto de operaciones, para la } \\
\text { elaboración de materiales bibliográficos }\end{array}$ & $\begin{array}{l}\text { A. Desarrollo alto. Profesores que dominan un } \\
\text { conjunto de operaciones para la elaboración de } \\
\text { materiales bibliográficos. } \\
\text { M. Desarrollo medio. Profesores que dominan } \\
\text { algunas operaciones para la elaboración de } \\
\text { materiales bibliográficos. } \\
\text { B. Desarrollo bajo. Profesores que no dominan } \\
\text { operaciones para la elaboración de materiales } \\
\text { bibliográficos. }\end{array}$ \\
\hline Motivación & $\begin{array}{l}\text { Voluntad para escribir materiales bibliográficos } \\
\text { condicionada por la necesidad que existe en el } \\
\text { estudiantado universitario }\end{array}$ & $\begin{array}{l}\text { A. Motivación alta. Profesores que demuestran } \\
\text { implicación personal con la elaboración de } \\
\text { materiales bibliográficos con un elevado } \\
\text { compromiso afectivo y disposición para actuar } \\
\text { con dicho contenido reconociendo su necesidad. } \\
\text { M. Motivación media. Profesores que demuestran } \\
\text { implicación personal con la elaboración de } \\
\text { materiales bibliográficos con un elevado } \\
\text { compromiso afectivo y disposición para actuar } \\
\text { con dicho contenido porque es necesario. } \\
\text { B. Motivación baja. Profesores que demuestran no } \\
\text { estar implicados con la elaboración de materiales } \\
\text { bibliográficos y consideran que no es necesario. }\end{array}$ \\
\hline
\end{tabular}

Frecuencia de Observación:

La observación realizada se ejecutó por tres semanas, en horario laboral, comprendido desde las 8:00 AM hasta las 5:30 PM, coincidente con la ejecución del sistema de trabajo del centro de estudio 


\section{Anexo 3. Entrevista a profesores}

Objetivo: Determinar la preparación que poseen los profesores para escribir los materiales bibliográficos

Compañero (a):

En el marco de una investigación relacionada con la necesidad de que los profesores escriban sus materiales bibliográficos, se necesita determinar la preparación que poseen los profesores para escribir estos materiales. Usted podría ayudar en tales empeños, a partir de su vivencia y actor principal al conocer la situación real que presenta la bibliografía en la carrera, por lo que le agradeceríamos que respondiera las siguientes preguntas:

1. ¿Cómo valora la diversidad de bibliografía con la que cuentan los estudiantes para contribuir a su formación en la asignatura Dirección Científica Educacional?

2. ¿Cuáles son los principales logros e insuficiencias que usted aprecia en las fuentes bibliográficas para abordar la DCE?

3. ¿En qué medida los profesores pueden contribuir a mejorar la bibliografía de sus alumnos?

4. ¿Pudiera precisar las principales fortalezas y debilidades que usted presenta para escribir materiales bibliográficos para sus estudiantes?

5. ¿Qué necesita en términos de preparación para comenzar a escribir los materiales bibliográficos de sus estudiantes?

6. ¿Se siente motivado para escribir los materiales bibliográficos de sus estudiantes?

\section{Anexo 4. Guía de Encuestas}

Objetivo: Profundizar en la preparación que tienen los profesores de DCE para la actualización de la bibliografía de sus estudiantes para lograr una mayor y mejor formación del profesional universitario.

\section{ENCUESTA}

Estimado compañero: sus experiencias y sugerencias pueden ayudar a diagnosticar la preparación que poseen los profesores de DCE para escribir los materiales bibliográficos de los alumnos. Agradecemos de antemano su evaluación sobre el tema que se aborda debiendo responder con total sinceridad sobre los aspectos que se señalan. Responda marcando con una X o contestando según el caso.

1. ¿Conoce usted algunas técnicas para escribir los materiales bibliográficos? Enúncielas.

2. ¿Conoce los deberes que tienen los profesores universitarios en la actualización de la bibliografía de sus estudiantes? Sí__ No_

De ser positiva su respuesta, ¿cómo clasificaría el resultado de este proceso para su centro?

3. ¿Cómo clasificaría usted la preparación que tienen los profesores para escribir los materiales bibliográficos de sus alumnos? Alto Medio Bajo

4. Analice su preparación para escribir materiales bibliográficos y evalúe cada aspecto en (B)-Bien, (R)-Regular o (M)-Mal. Conocimientos: Habilidades: Motivación: Otros:

\section{Cómo citar este artículo:}

Martínez-Mollineda, Carlos, Viviana Hernández-Fernández y Mairelys Torres-Falcón (2018), “La elaboración de materiales bibliográficos para la formación del profesor universitario", en Revista Iberoamericana de Educación Superior (RIES), México, UNAM-IISUE/Universia, vol. IX, núm. 25, pp. 176-193, http://dx.doi.org/10.22201/ iisue.20072872e.2019.25.348 [consulta: fecha de última consulta]. 